\title{
Fast and broadband detector for laser radiation
}

Davide Scorticati, Giacomo Crapella, Sergio Pellegrino

Davide Scorticati, Giacomo Crapella, Sergio Pellegrino, "Fast and broadband detector for laser radiation," Proc. SPIE 10539, Photonic Instrumentation Engineering V, 105390I (22 February 2018); doi: 10.1117/12.2294002

SPIE. Event: SPIE OPTO, 2018, San Francisco, California, United States 


\title{
Fast and broadband detector for laser radiation
}

\author{
Davide Scorticati*, Giacomo Crapella, Sergio Pellegrino \\ Laserpoint srl, via Burona 51, Vimodrone (Mi), 20090, Italy \\ *scorticati@laserpoint.it
}

\begin{abstract}
We developed a fast detector (patent pending) based on the Laser Induced Transverse Voltage (LITV) effect. The advantage of detectors using the LITV effect over pyroelectric sensors and photodiodes for laser radiation measurements is the combination of an overall fast response time, broadband spectral acceptance, high saturation threshold to direct laser irradiation and the possibility to measure pulsed as well as cw-laser sources.

The detector is capable of measuring the energy of single laser pulses with repetition frequencies up to the MHz range, adding the possibility to also measure the output power of cw-lasers.

Moreover, the thermal nature of the sensor enables the capability to work in a broadband spectrum, from $\mathrm{UV}$ to $\mathrm{THz}$ as well as the possibility of operating in a broad-range $\left(10^{-3}-10^{2} \mathrm{~W} / \mathrm{cm}^{2}\right)$ of incident average optical power densities of the laser radiation, without the need of adopting optical filters nor other precautions.
\end{abstract}

Keywords: Fast, Broadband, Thermal, Detector, Laser, LITV

\section{INTRODUCTION}

Fast laser-radiation detectors nowadays are mainly represented by photodiodes, pyroelectric sensors and axial thermoelectric devices. Thermoelectric devices are subdivided into two different groups: the devices using the standard longitudinal thermoelectric effect induced at the junction of different types of materials and the devices based on the laser induced transverse voltage (LITV) effect.

The main advantage of photodiodes over other types of sensors is the fastest response timewise. Based on the recombination mechanism of the electron-hole pairs in P-N junctions, sub-nanosecond response time can be achieved by devices utilizing this physical principle. Moreover, the high sensitivity of those devices allows the measurement of extremely low power continuous wave (cw) laser beams and single laser pulses. By contrast, the high sensitivity is combined with a low saturation threshold while measuring laser-radiation. The response of photodiodes suffers of spatial non-uniformity and the strong dependence on the operating temperature as well as on the wavelength of the impinging radiation. Besides, the spectral working band is physically limited by the energy-gap of the semiconductors in use.

Sensors based on the pyroelectric effect transduce a thermal gradient into an electric signal. That is, the polarization change due to a thermal gradient gives rise to a voltage across the crystal. On the one hand, pyroelectric sensors do have the advantage over photodiodes to be broadband (i.e. from UV to THz) benefiting from their thermal nature. Besides, they also combine the high sensitivity (which can be higher or equal to $1 \mathrm{kV} / \mathrm{W}$ ) with the possibility to be tailored in their design for high energy pulses. On the other hand, pyroelectric sensors are limited to relatively low laser repetition rates (currently up to $200 \mathrm{kHz}$ ) and the inability to measure continuous wave (cw) laser radiation, due to the natural leakage current across the active material. In fact, the working principle of pyroelectric materials, based on the response to transient changes of temperature, allows just measurements of pulsed to quasi-cw, but not purely $\mathrm{cw}$ or long pulsed laser sources.

Sensors based on the standard longitudinal thermoelectric effect are commonly designed adopting multiple electrically interconnected thermocouples, which can measure a heat flux axially across a suitable substrate [1]. This type of sensors using the standard thermoelectric effect are an evolution of the common radial thermopile design. Working on a thermal principle, the spectral acceptance region of this kind of sensors is still broadband. However, the thermal design of this kind of sensors only allows for relatively slow response times (currently greater than $100 \mathrm{~ms}$ ). Moreover, the design of multiple axial thermocouples often implies a scarce coverage of the active area of the sensor.

Sensors using the laser induced transverse voltage (LITV) effect also transduce a thermal gradient into an electric signal. Thin films of suitable materials, deposited with a slant angle between the evaporation source and the substrate are known to show a transverse thermoelectric response to laser-irradiation. That is, if a thermal gradient is present along the

\footnotetext{
Photonic Instrumentation Engineering V, edited by Yakov G. Soskind,

Craig Olson, Proc. of SPIE Vol. 10539, 105390I - (C) 2018 SPIE

CCC code: $0277-786 \mathrm{X} / 18 / \$ 18 \cdot$ doi: $10.1117 / 12.2294002$
} 
normal direction to the film surface, a thermoelectric response is generated, longitudinally to the plane of the film surface. The intensity of the generated electric signal, extractable along the longitudinal plane, has a well-defined direction and it is parallel to the projection of the angled grains of the thin film active material grown onto the substrate, showing a minimum in the coplanar perpendicular direction. [2-4]

The adoption of the LITV effect has the intrinsic advantage of showing a good conversion efficiency of a thermal signal into an electric voltage, while showing response times in the nanosecond timescale. Besides, the fabrication of the device results more controllable as it requires less technological steps, hence resulting cheaper and simplified. Another advantage of the LITV based devices over standard thermoelectric devices is the uniform coverage of the active area, with respect to a design based on axially disposed thermocouples.

The advantage of sensors using the LITV effect over pyroelectric sensors and photodiodes for laser radiation measurement is the combination of an overall fast response time, broadband spectral acceptance, high saturation threshold to direct laser irradiation and the possibility to measure pulsed as well as cw-laser sources. Moreover, the active area of LITV based sensors does not have limitations in size, compared to fast photodiodes and pyroelectric sensors, whereas the uniformity of the deposition of the active film is maintained.

Although sensors based on the LITV effect are very promising in the technological area of laser radiation energy/power sensing, a limitation is posed by the disadvantageous requirement of complex alloys -often composed by toxic materialsfor the production of the active layer [3-4]. Moreover, some of those detectors adopt a multilayered structure of the active materials, adding complexity to the manufacturing process flow [5]. Besides, between the active layer and the substrate an additional layer with an oriented grain structure is required, which influences the formation of tilted grains during the deposition of the active material, adding a supplementary and critical processing step [4].

In this work, we characterize a fast detector of laser radiation (patent pending) based on the LITV effect for the measurement of direct laser radiation, which is easy to produce, reliable and free of hazardous materials.

\section{EXPERIMENTAL METHOD}

\subsection{Laser}

Measurements were performed using pulsed laser with variable pulse duration (i.e. in the range 4-200 ns), repetition frequency (i.e. from $20 \mathrm{kHz}$ to $1 \mathrm{MHz}$ ), max pulse energy of $1 \mathrm{~mJ}, 1064 \mathrm{~nm}$ wavelength and maximum average output power of $20 \mathrm{~W}$.

Measurements of cw-laser radiation were also performed using a $1064 \mathrm{~nm}$ wavelength source.

\subsection{Optical measurements}

Optical measurements were performed using a spectrophotometer equipped with an integrating sphere in the range 0.25$1.1 \mu \mathrm{m}$.

\subsection{Electrical measurements}

Electrical measurements were performed adopting an oscilloscope with a maximum sampling rate of $40 \mathrm{GS} / \mathrm{s}$ and $1 \mathrm{GHz}$ bandwidth, using a differential probe.

\section{RESULTS AND DISCUSSION}

\subsection{Response}

The sensor acts as an electromotive force (e.m.f.) generator when irradiated by laser radiation. Its signal is proportional to the temperature gradient formed between the external surface of the active film and its interface with the substrate.

With respect to figure 1, the response of the sensor can be expressed by the formula:

$$
\mathrm{V}(\mathrm{t}) \propto \sin (2 \alpha) \cdot(\mathrm{S} \|-\mathrm{S} \perp) \cdot \Delta \mathrm{T}(\mathrm{t})
$$

where $\alpha, \mathrm{S} \|, \mathrm{S} \perp$ and $\Delta \mathrm{T}$ are, respectively, the tilt-angle of the grains of the active layer 2 with respect to the normal direction to the substrate plane, the Seebeck coefficient in the direction parallel to the projection of the angled grains of 
the active layer 2 onto the substrate surface 1, the Seebeck coefficient perpendicular to the substrate surface and the temperature gradient across the film.

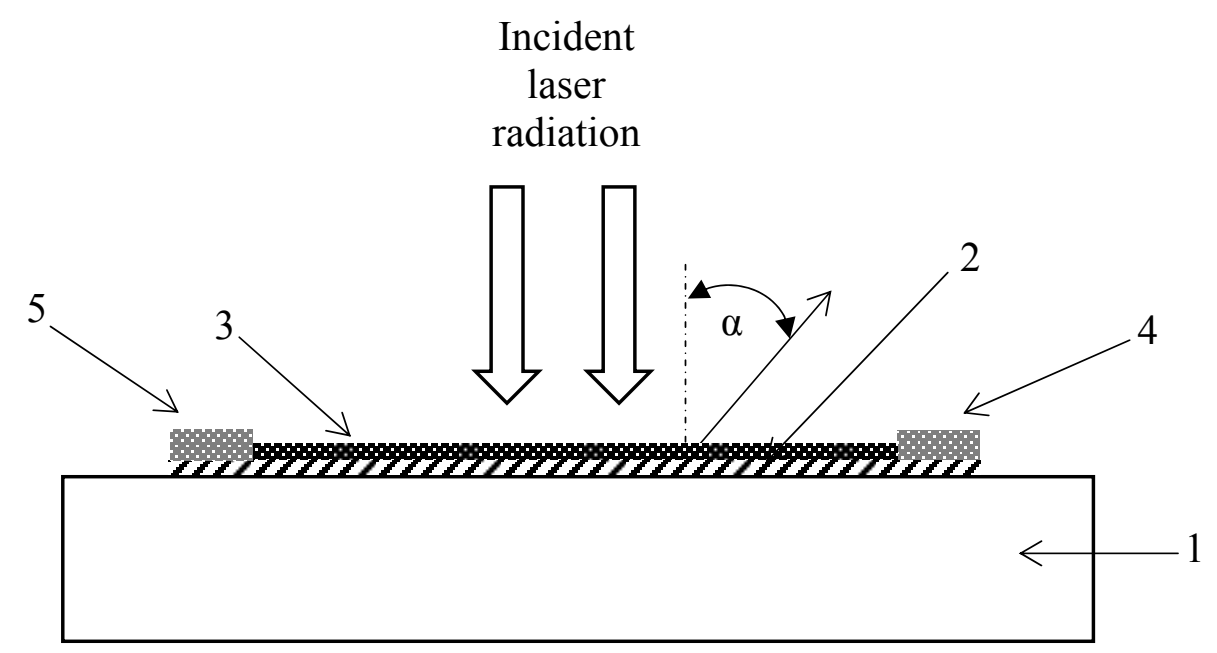

Fig. 1 Cross section of the sensor. 1) is the substrate material, 2) the active layer, 3) the absorber layer, 4) and 5) the electrodes.

\subsection{Footprint and active area}

The footprint of the packaged detector is $33 \times 36 \times 7 \mathrm{~mm}$, with an active area of $15 \times 15 \mathrm{~mm}$.

Depending on sizing of the used heat-sink, the detector can operate in a different range of optical power of the incident laser beam.

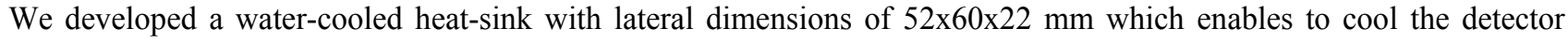
down with minimal footprint for an easy integration of the detector into laser systems.

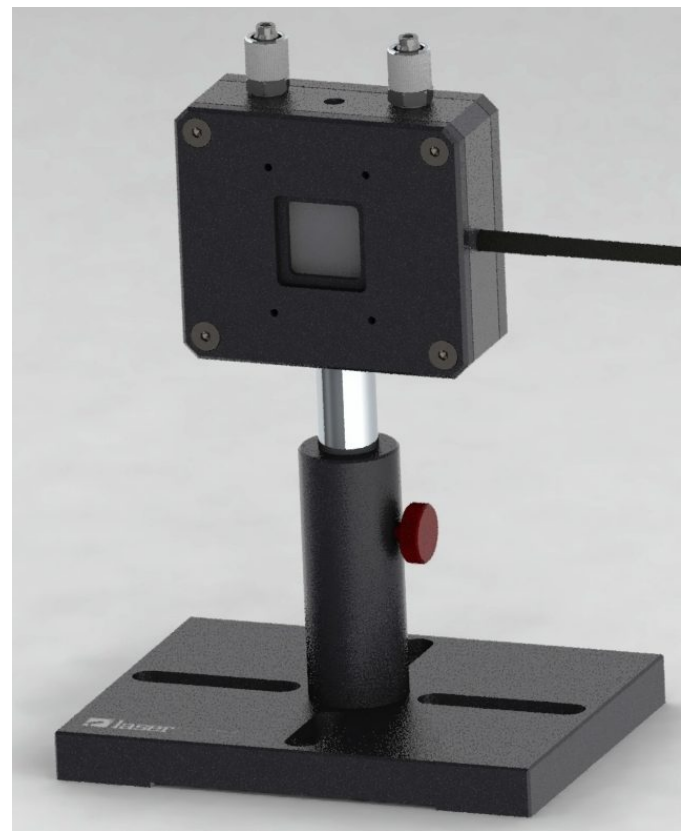

Fig. 2 Picture of the complete detector head, showing the detector mounted onto the water-cooled heat-sink. 


\subsection{Linearity of the response vs power}

The response of the sensor under cw-laser radiation, up to about $50 \mathrm{~W}$, is depicted in Fig. 3. Data show the output signal of the detector without any post-linearization. The plotted line indicates the linear fit, which quantifies the linearity of the detector within the measured range with an R coefficient of 0.9998. Having a good linearity is crucial for the calibration of the detector once manufactured.

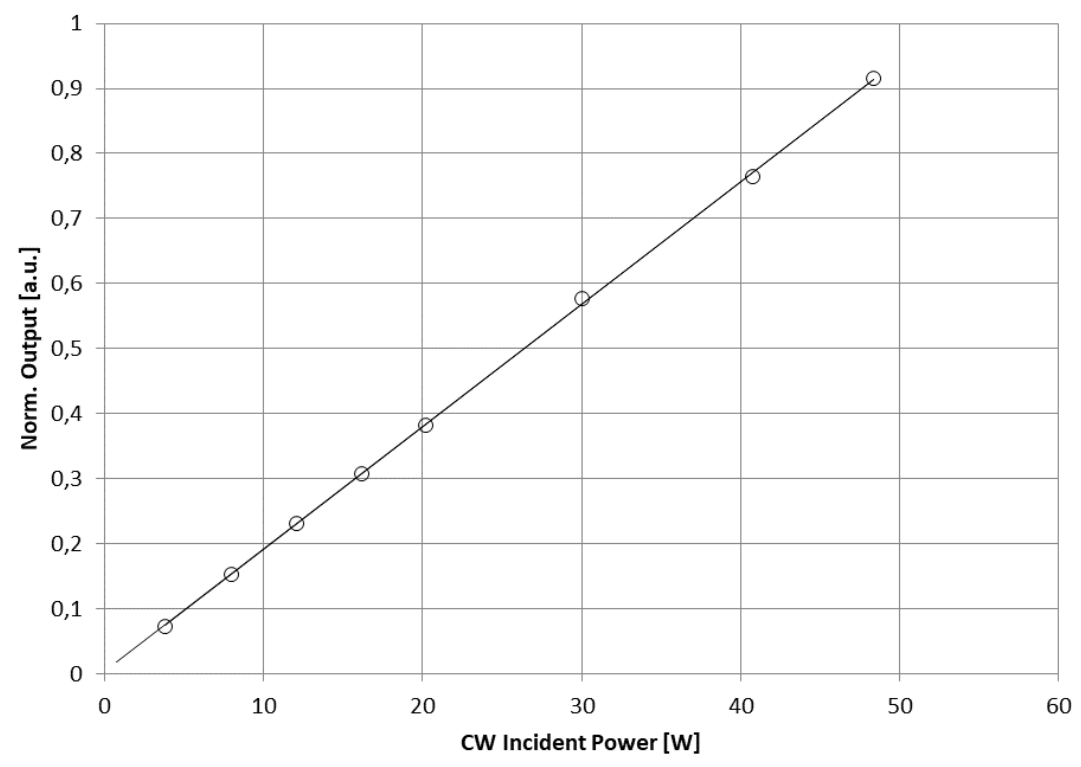

Fig. 3 Response of the detector vs. power of cw-laser source.

The range of linearity is preserved -and can possibly be extended- as long as two conditions are fulfilled. First, the laser fluence must be lower than the damage threshold at the surface of the detector. Second, the heat sink is design to dissipate the absorbed laser power, allowing a maximum operating temperature of $80^{\circ} \mathrm{C}$.

Currently, the maximum allowed incident power level is above $100 \mathrm{~W}$.

\subsection{Linearity of the response vs Pulse energy}

The response of the detector to pulses of different energy is shown in fig. 4. In the same figure, it is also depicted the independency of the energy measurements from the pulse duration $\tau_{\mathrm{p}}$, which in turn indicates the independency of the measurements from power density. 


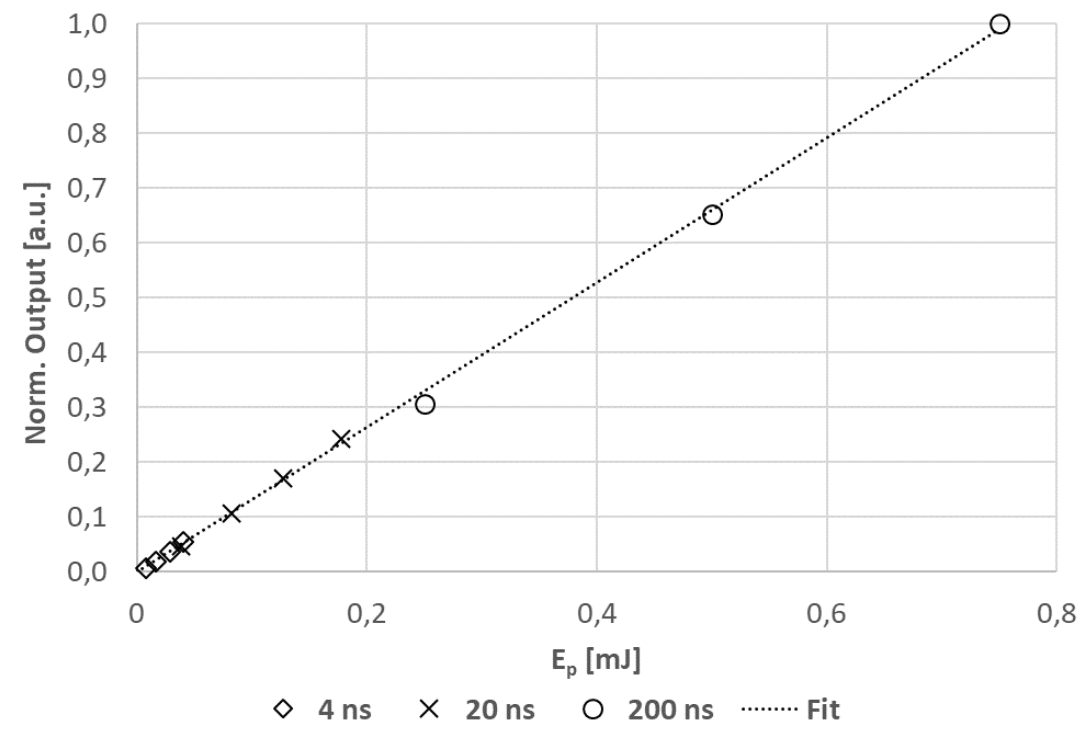

Fig. 4 Response of the detector vs. energy of the incident pulses with different pulse durations.

The measurements of the energy of the incident pulses $E_{\mathrm{p}}$ is independent from the spot size as well and the energy range of the detected pulses ranges from $10 \mathrm{uJ}$ up to tens of $\mathrm{mJ}$.

\subsection{Stability of the response over the whole active area}

The active area of the detector is a square of side $15 \mathrm{~mm}$. Scanning a laser beam across a matrix of points along the whole active area, the response of the sensor was maintained within $<1 \%$ accuracy.

\subsection{Response time}

Different definitions can be used for the response time. We use here the $0-100 \%\left(\tau_{0-100}\right)$ response time definition, which is the time needed by the detector to restore the signal output to the level of the baseline, after the incident laser pulse is terminated. Such definition was chosen because $f_{\max }=1 / \tau_{0-100}$ defines the maximum measurable frequency of a train of laser pulses by the detector.

As the voltage output signal of the detector is directly proportional to the thermal gradient across the active film thickness, see equation 1 , it is possible to tailor the response time $\tau_{0-100}$ of the device by adjusting the thickness of the film. On the other hand, a thinner active film also decreases the maximum output voltage of the detector, when the duration of the incident laser pulses $\tau_{\mathrm{p}}$ is longer than the thermal time needed to restore thermal equilibrium across the film.

The thickness of the active film was finally set in order to achieve a maximum operating frequency of $1 \mathrm{MHz}$ for laser pulses with pulse durations up to $200 \mathrm{~ns}$. That is, the sensor is able to detect single laser pulses up to $1 \mathrm{MHz}$ of frequency, for pulses with $\tau_{\mathrm{P}}$ up to $200 \mathrm{~ns}$, i.e. with a duty of 0.2 .

In fig. 5 , it is depicted the fast response of the detector to a train of laser pulses ( $\tau_{\mathrm{P}}=4 \mathrm{~ns}$ ) with a repetition rate of 1 MHz. 


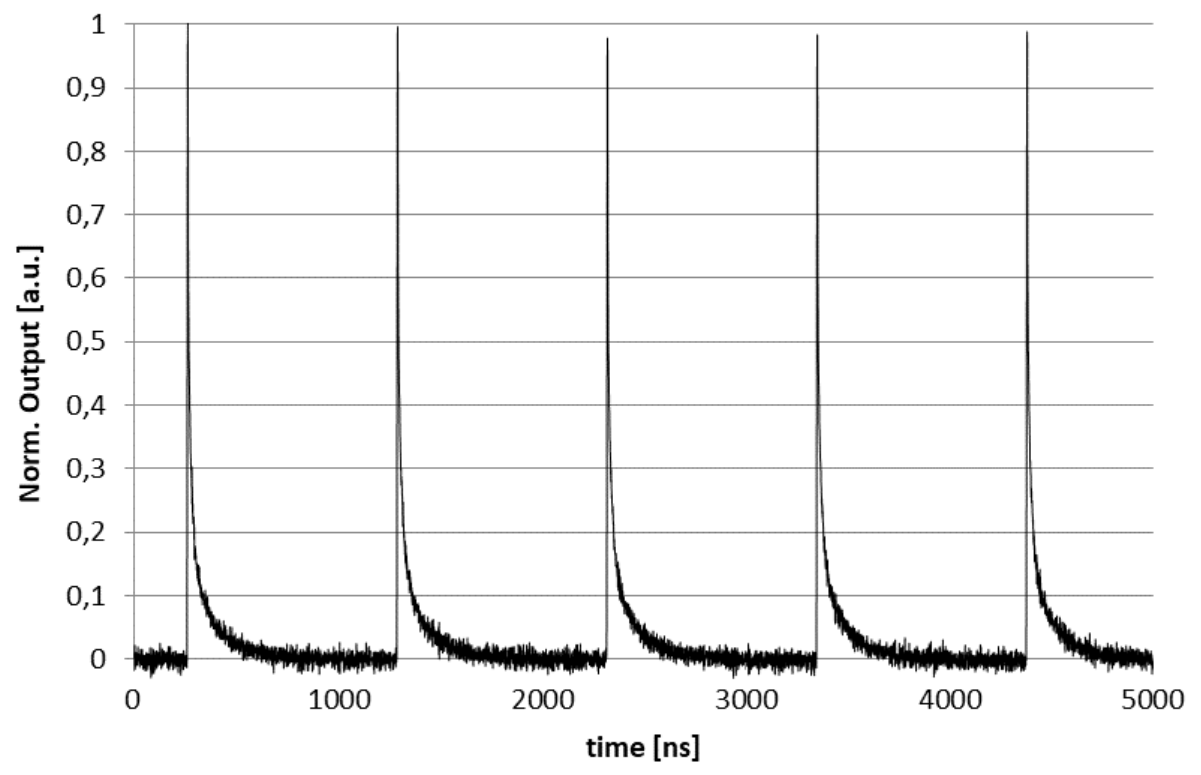

Fig. 5 graph of the output voltage signal as a function of time for the detector in response to irradiation by a train of pulses with $\tau_{\mathrm{P}}$ of $4 \mathrm{~ns}$ at $1 \mathrm{MHz}$ repetition frequency.

\subsection{Damage threshold}

As visible in fig. 1, a coating is applied onto the active film. The coating acts both as the absorber of the incident laser light, as well as a passivation layer for the active film, otherwise in contact with the atmosphere.

A thicker coating with higher damage threshold compared to the one of the active film, results in an increased damage threshold for the detector. However, a thicker coating also increases the thermal resistance of the system, hence increasing the response time.

It was found that the damage threshold of the detector in its fastest configuration, i.e. with $1 \mathrm{MHz}$ bandwidth, was greater than $30 \mathrm{~mJ} / \mathrm{cm}^{2} /$ pulse for pulses of $200 \mathrm{~ns}$.

\subsection{Optical acceptance}

The thermal nature of the LITV effect allows the detector to work in a broadband wavelength range of incident radiation, as long as the electromagnetic radiation is absorbed and transformed into heat by the absorption coating.

An important characteristic of the coating is its spectral flatness, which is of great importance for a simple calibration of the detector. The optical reflectance of the detector is depicted in Fig. 6. Within the whole measurement range, i.e. from $250 \mathrm{~nm}$ to $1100 \mathrm{~nm}$, the reflectance curve shows a smooth and overall relatively flat behavior. 


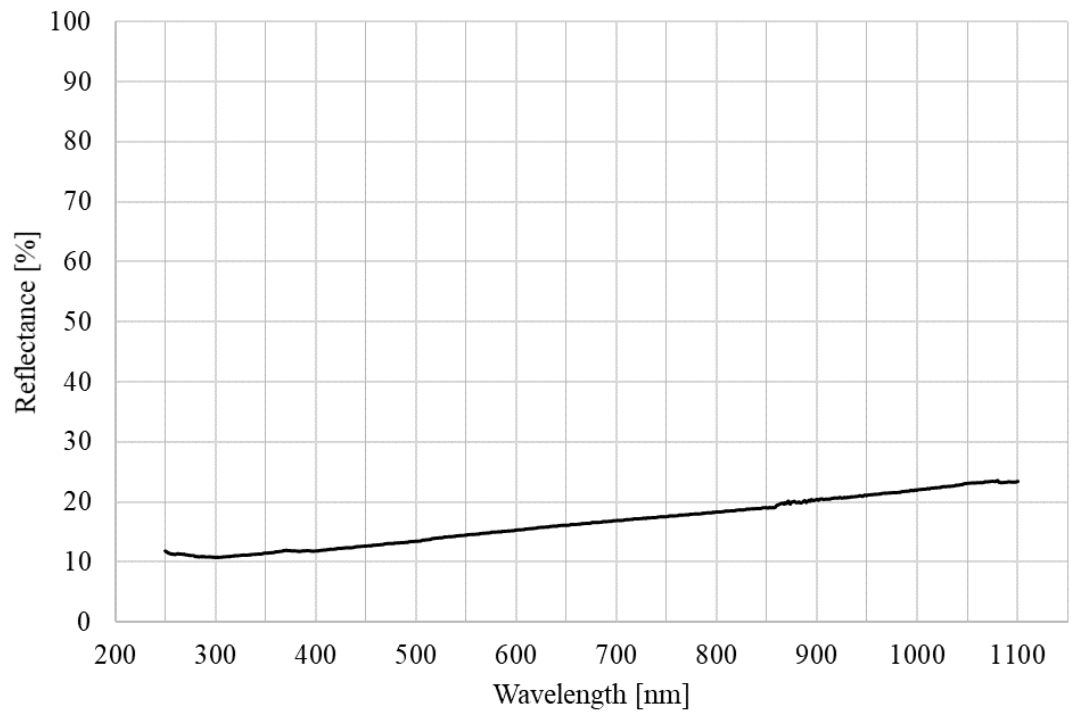

Fig. 6 Optical reflectance of the surface of the detector in the range from $250 \mathrm{~nm}$ to $1100 \mathrm{~nm}$.

\section{CONCLUSIONS}

Our patent pending detector is capable of measuring the energy of single laser pulses in the range of from $10 \mathrm{uJ}$ up to tens of $\mathrm{mJ}$ with repetition frequencies up to the $\mathrm{MHz}$ range, adding the possibility to also measure the output power of cw-lasers.

Moreover, the thermal nature of the sensor enables the capability to work in a broadband spectrum, from UV to THz as well as the possibility of operating in a broad-range $\left(10^{-3}-10^{2} \mathrm{~W} / \mathrm{cm}^{2}\right)$ of incident optical power densities of the laser radiation, without the need of adopting optical filters or other precautions.

The linearity of the response of the detector to power and energy of the incident pulses are warrantee of an easy calibration procedure, together with the overall smooth behavior of the reflectance showed by the applied coating.

In the fastest configuration possible, for pulses of $200 \mathrm{~ns}$, the damage threshold is currently greater than 30 $\mathrm{mJ} / \mathrm{cm}^{2} /$ pulse. Finally, the active area is of $15 \times 15 \mathrm{~mm}$ and the compact footprint of the detector allows an easy integration of the latter into laser systems.

\section{REFERENCES}

[1] S. Dröscher, M. Zahner, E. Schwyter, T. Helbling, C. Hierold, Reinventing Thermal Laser Power Measurements, Lasers in Manufacturing Conference (2015).

[2] Zhang, GY., Zhang, H., Tan, SL. et al., A novel strongly correlated electronic thin-film laser energy/power meter based on anisotropic Seebeck effect, Appl. Phys. A 116: 1033 (2014).

[3] Song, S., Yu, L., Hu, J. et al., Laser-induced transverse voltage effect and thermopower anisotropy of c-axis inclined $\mathrm{Ca}_{3} \mathrm{Co}_{4} \mathrm{O}_{9}$ thin film, Appl. Phys. A 123: 595 (2017).

[4] P.X.Zhang, C.Wang, S.L.Tan, H.Zhang, H.-U.Habermeier, Improving the performance of thermoelectric devices by doping Ag in LaPbMnO3 thin films, Journal of Crystal Growth, Volume 310, Issue 11, Pages 2732-2737 (2008).

[5] Fischer, K., Stoiber, C., Kyarad, A. et al., Anisotropic thermopower in tilted metallic multilayer structures, Appl. Phys. A 78: 323 (2004). 